\title{
Characterization of Ketoprofen/Methyl- $\beta$-Cyclodextrin Complexes Prepared Using Supercritical Carbon Dioxide
}

\author{
Mauro Banchero, Silvia Ronchetti, and Luigi Manna \\ Dipartimento Scienza Applicata e Tecnologia, Politecnico di Torino, Corso Duca degli Abruzzi 24, 10129 Torino, Italy \\ Correspondence should be addressed to Mauro Banchero; mauro.banchero@polito.it
}

Received 11 February 2013; Accepted 25 March 2013

Academic Editor: Ignacio Gracia Fernández

Copyright ( 2013 Mauro Banchero et al. This is an open access article distributed under the Creative Commons Attribution License, which permits unrestricted use, distribution, and reproduction in any medium, provided the original work is properly cited.

Complexes of methyl- $\beta$-cyclodextrin and ketoprofen, a crystalline anti-inflammatory drug with poor water solubility, have been prepared for the first time in the presence of supercritical $\mathrm{CO}_{2}$ at $40^{\circ} \mathrm{C}$ and $20 \mathrm{MPa}$. The supercritical treatment allows these pharmaceutical formulations to be prepared without the use of any auxiliary agents or organic solvents. The treated samples were characterized through differential scanning calorimetry, X-ray diffractometry, and the Fourier transform infrared spectroscopy to exclude the presence of crystalline drug and check the formation of the complexes. The increase of the drug dissolution rate was investigated performing in vitro release tests in aqueous solutions. The results showed that the supercritical treatment can be an efficient method to obtain inclusion complexes with enhanced release kinetics. The operating methods of the release tests, that is, the "tablet method" or the "dispersed amount method," affected both the dissolution rate and its dependence on the drug amount in the samples. On the contrary, the variation of the $\mathrm{pH}$ of the dissolution medium did not show any effect on the release rate of the supercritical complexes.

\section{Introduction}

Supercritical fluid technology has recently emerged as a green and novel technique for various processes in the pharmaceutical area [1]. This is connected to the specific features of dense gases such as high compressibility and diffusivity, very high evaporation rate and "tunable" solvent power, which can be varied through density modulation by simply changing either the pressure or the temperature [2]. Carbon dioxide is the most used supercritical fluid thanks to its low critical point $\left(31.2^{\circ} \mathrm{C}, 7.4 \mathrm{MPa}\right)$, inexpensiveness, nonflammability, nontoxicity, recyclability, and environmental benignity [2]. It can be used as a solvent, antisolvent, or processing aid in many pharmaceutical operations such as particle generation and micronization, coating, foaming, tissue engineering, extrusion, sterilization, or inclusion complexation with cyclodextrins [1-3].

Cyclodextrins are cyclic oligosaccharides composed of $(\alpha-1,4)$-linked $\alpha$-D-glucopyranose units, whose molecules are characterized to have a hydrophilic external surface and a lipophilic internal cavity. In fact, the chair conformation of the glucopyranose units makes cyclodextrin molecules have the shape of a truncated cone with a central cavity. The external surface is hydrophilic because of the presence of hydroxyl groups, which are oriented towards the cone exterior with the primary hydroxyl groups at the narrow edge of the cone and the secondary ones at the wider edge. The presence of the skeletal carbons and ethereal oxygens of the glucose residues gives a lipophilic character to the central cavity. This type of structure provides to the cyclodextrin the ability to form water-soluble inclusion complexes with guest hydrophobic molecules of suitable size [4].

There are three main naturally occurring cyclodextrins: $\alpha$-, $\beta$-, and $\gamma$-cyclodextrin composed of six, seven, and eight glucopyranose units, respectively. $\beta$-cyclodextrin (Figure 1) is the most used natural cyclodextrin in the pharmaceutical field since it is available in pure form and has a relatively low cost [5]. Its cavity size is appropriate to form inclusion complexes with many poorly soluble drug molecules [6], and this results in favourable changes in the physicochemical properties of the guest molecule, such as increased solubility, enhanced dissolution rate, better stability, and 


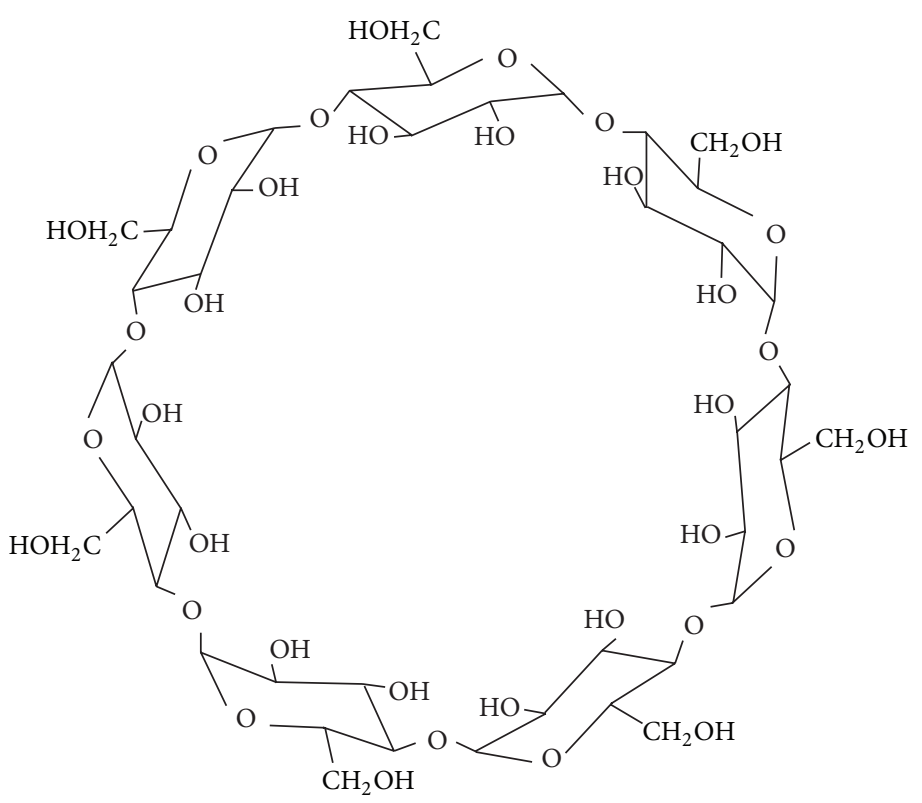

(a)

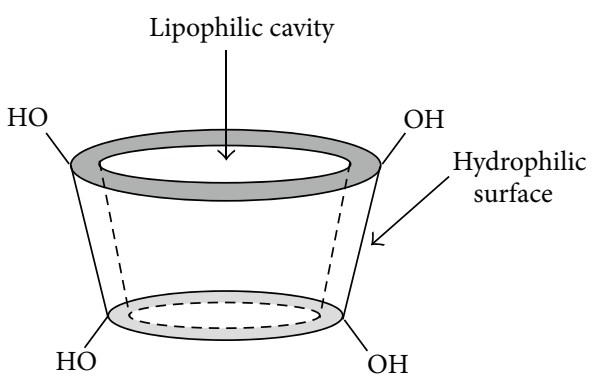

(b)

FIgURE 1: (a) The chemical structure and (b) the toroidal shape of $\beta$-cyclodextrin.

improved organoleptic characteristics [7]. In addition to natural cyclodextrins, many chemically modified derivatives are commercially available. These are obtained by substituting some of the hydroxyl groups on the external surface with other functional groups (hydroxypropyl, methyl, sulfobuty lether, etc.), and this results in improved aqueous solubility [8].

The production of pharmaceutical formulations that contain cyclodextrin inclusion complexes can be obtained through many conventional techniques, such as kneading, spray drying, freeze drying, and coprecipitation from various solvents. However, many of these methods suffer from some limitations, such as long process time, low efficiency for inclusion complexation, the need of additional drying step, and high residual organic solvent content [9]. The use of supercritical carbon dioxide $\left(\mathrm{scCO}_{2}\right)$ as an unconventional complexation medium can be an appealing alternative thanks to the unique properties that were mentioned above. In particular, carbon dioxide is an ideal substitute for organic solvents thanks to the lack of toxicity in the final product since there is no residual solvent after depressurization. Furthermore, the excellent mass transfer properties and high solvent power of this fluid render this technique suitable for the complexation of thermally labile compounds [2].

Several trials of $\mathrm{drug} / \beta$-cyclodextrin complexation through $\mathrm{scCO}_{2}$ can be found in the literature [10-18], while other works can be found as far as the use of chemically modified cyclodextrins is concerned [6,19-23]. Even though alternative techniques were developed $[9,24,25]$, the most used one consists in keeping a physical mixture of the drug and cyclodextrin in static contact with $\mathrm{scCO}_{2}$ at constant temperature and pressure and for a fixed period of time. In order to enhance the inclusion efficiency, some experiments were also conducted after adding water and/or auxiliary agents to the initial physical mixtures [11, 14, 16, 23, 26, 27] or modifying the supercritical medium through the addition of cosolvents [28].

The use of methyl- $\beta$-cyclodextrin $(\mathrm{Me} \beta \mathrm{CD})$ as the host molecule for drug inclusion complexation through $\mathrm{scCO}_{2}$ has, perhaps, given the most promising results among all the investigated systems $[19,21,22]$. First, it does not require any water or auxiliary agents to improve the inclusion efficiency since it was found that $\mathrm{Me} \beta \mathrm{CD}$ is liquefied under the working conditions of the supercritical process $[19,21,22]$, which would help the drug molecules better reach the cyclodextrin cavity [21]. Second, the obtained drug complexes show almost instantaneous dissolution profiles in aqueous solutions since the solubility of $\mathrm{Me} \beta \mathrm{CD}$ in water at room temperature is much higher $(>2000 \mathrm{mg} / \mathrm{mL})$ than that of the unsubstituted $\beta$-cyclodextrin (18.5 mg/mL) [19].

Ketoprofen (KP) is one of the most used nonsteroidal anti-inflammatory drugs. It is a crystalline acidic drug with poor water solubility, and its complexation with natural and chemically modified cyclodextrins through conventional techniques has been widely investigated [29-31]. Even though it was established that $\mathrm{Me} \beta \mathrm{CD}$ is the most effective carrier to increase the dissolution properties of KP independently of the preparation technique [29-31], this drug/cyclodextrin system has never been tested in the presence of a supercritical solvent. The previous literature works only examined the supercritical complexation of KP either with the natural $\beta$ cyclodextrin [14, 27] or its hydroxypropyl derivative [27] and pointed out that the addition of water and/or an auxiliary agent, the L-lysine, is required to achieve a high complexation efficiency. 
<smiles>CC(C(=O)O)c1cccc(C(=O)c2ccccc2)c1</smiles>

(a)

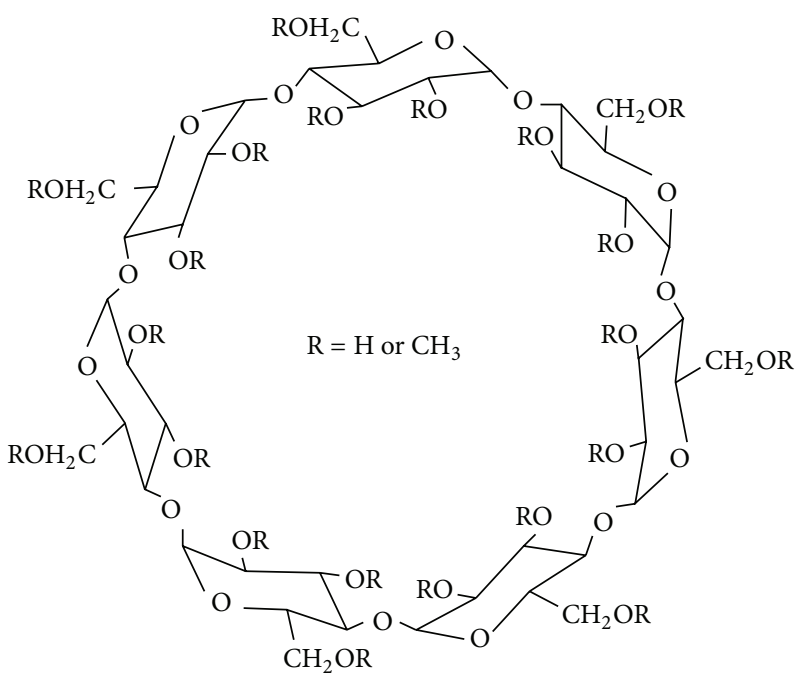

(b)

FIGURE 2: (a) KP chemical structure. (b) Me $\beta \mathrm{CD}$ chemical structure with average $\mathrm{MW} \sim 1310$; the number of $\mathrm{R}=$ methyl functions corresponds to 1.6-2 random substitutions per each anhydrous glucose unit (i.e., 11.2-14 substitutions per each cyclodextrin molecule).

In this work, the supercritical complexation of $\mathrm{Me} \beta \mathrm{CD}$ with ketoprofen (KP) has been investigated for the first time. The working conditions of the supercritical treatment were fixed at $40^{\circ} \mathrm{C}$ and 200 bar on the basis of a previous literature work with the same cyclodextrin [19], and the experiments were conducted treating physical mixtures with different drug contents without the addition of water or any auxiliary agents. The characterization of the treated samples was carried out through differential scanning calorimetry (DSC), X-ray diffractometry (XRD), and the Fourier transform infrared (FTIR) spectroscopy. Subsequently, the in vitro release profiles of the treated samples were determined in an aqueous solution and were compared with those of the physical mixtures with the same drug content.

\section{Materials and Methods}

2.1. Materials. The $\mathrm{CO}_{2}(99.998 \%)$ was purchased from SIAD S.p.A. (Italy). The ketoprofen $\left(\geq 98 \%\right.$, melting point $\sim 94^{\circ} \mathrm{C}$ ) and methyl- $\beta$-cyclodextrin $(\geq 98 \%$, average $\mathrm{MW} \sim 1310$, melting point $\sim 180-182^{\circ} \mathrm{C}$ ) were supplied by Sigma-Aldrich. The chemical structures of $\mathrm{KP}$ and $\mathrm{Me} \beta \mathrm{CD}$ are reported in Figure 2.

2.2. Preparation of the Inclusion Complexes through $\mathrm{scCO}_{2}$. The supercritical process was carried out in a static mode to achieve the inclusion of the drug in the cyclodextrin. The apparatus is equipped with a volumetric pump, a $50 \mathrm{~mL}$ vessel, and a depressurisation system. The vessel is positioned inside an oven, which maintained all the system at constant temperature. Further details of the apparatus can be found elsewhere [32-34].

Approximately, $1 \mathrm{~g}$ of the physical mixtures of the compounds were introduced into filter paper cylinders that were set inside the vessel. The apparatus was operated in
TABLE 1: Composition of the physical mixtures.

\begin{tabular}{lcc}
\hline Physical mixture & Criterion & $\begin{array}{c}\text { KP content } \\
(\% \text { w/w })\end{array}$ \\
\hline A & $\begin{array}{c}\text { Equimolar content of KP and } \\
\text { Me } \beta \text { CD }\end{array}$ & 16.3 \\
B & $\begin{array}{c}15 \% \text { excess of KP with respect to } \\
\text { the equimolar content }\end{array}$ & 18.7 \\
C & $\begin{array}{c}15 \% \text { defect of KP with respect to } \\
\text { the equimolar content }\end{array}$ & 13.8 \\
\hline
\end{tabular}

a static mode, and the samples were maintained in $\mathrm{scCO}_{2}$ atmosphere at constant temperature and pressure for a fixed period of time. At the end of the process, the vessel was depressurized and each sample was analysed.

The working conditions of the supercritical treatment were fixed at $40^{\circ} \mathrm{C}$ and 200 bar with a contact time of $1 \mathrm{~h}$. These conditions assured that $\mathrm{Me} \beta \mathrm{CD}$ was melted and were similar to those adopted by Charoenchaitrakool and coworkers with ibuprofen and $\mathrm{Me} \beta \mathrm{CD}$ [19].

All the physical mixtures were prepared by manually mixing the proper components in a mortar, and their compositions are reported in Table 1. Physical mixture A was prepared with a molar ratio between $\mathrm{KP}$ and $\mathrm{Me} \beta \mathrm{CD}$ equal to $1: 1$ as was suggested by a previous work where $\mathrm{KP} / \mathrm{Me} \beta \mathrm{CD}$ complexes were prepared through conventional techniques [30]. Mixtures B and C were prepared introducing a $15 \%$ excess and a $15 \%$ defect of $\mathrm{KP}$ with respect to the equimolar content of mixture A.

2.3. DSC Analyses. Differential scanning calorimetry was performed in a METTLER DSC30 instrument on $5 \mathrm{mg}$ samples positioned in aluminium pans by increasing the temperature from 30 to $120^{\circ} \mathrm{C}$ at a heating rate of $10^{\circ} \mathrm{C} / \mathrm{min}$ under a $\mathrm{N}_{2}$ gas stream. 
2.4. XRD Analyses. Powder XRD analyses were carried out to verify the absence of crystalline structure in the treated samples using a Philips X'Pert-MPD diffractometer (Cuk $\alpha$ radiation; $2 \theta$ range $4-50^{\circ} ; \Delta 2 \theta$ step: $0.02^{\circ}$; step time $1 \mathrm{~s}$ ), with a fixed specimen and moving the X-ray source and detector simultaneously by a $\theta$ angle.

2.5. FTIR Spectroscopy. FTIR analysis was performed in a Thermo-Nicolet 5700 FTIR spectrophotometer equipped with a mercury-cadmium-telluride detector, which was cooled with liquid nitrogen. The powder (3 mg) was combined with potassium bromide $(180 \mathrm{mg})$, pressed into a thin glass pellet, and then analysed using 256 scans with a $1 \mathrm{~cm}^{-1}$ resolution step.

2.6. In Vitro Release Tests. In vitro release tests of the obtained complexes were compared with those of the physical mixtures with the same drug content. Both the treated samples and physical mixtures were compressed into $10 \mathrm{~mm}$ wide and a few millimetres thick cylindrical tablets using a manual press (Speac Inc., USA). The weight of each tablet was $100 \mathrm{mg}$, and the drug dose varied depending on the KP content in the impregnated or physical mixture powders. The compression pressure was set at 1 tonne, and the compression chamber was connected to an ejector to prevent air from being trapped in the tablet. The release tests were performed with cylindrical tablets in order to minimize the effect of particle size distribution. Anyway, some tests were also performed directly using $100 \mathrm{mg}$ of the powders of the physical mixtures or of the treated samples without producing any tablets.

The release tests were carried out in a USPII apparatus (Erweka Dissolution Tester, GmbH, Germany) equipped with a paddle stirrer rotating at $50 \mathrm{rpm}$ and an on-line UV spectrophotometer (UNICAM UV2-300). The vessel contained a $750 \mathrm{~mL}$ solution of $\mathrm{HCl}$ in distilled water at $37^{\circ} \mathrm{C}$ and $\mathrm{pH} 2$. Some experiments were also performed with a solution at $\mathrm{pH}$ 6 to investigate if the drug release was affected by the value of the $\mathrm{pH}$. The drug release profiles were measured detecting the absorbance at $259 \mathrm{~nm}$.

\section{Results and Discussion}

The aim of the supercritical treatment is to produce drug/ cyclodextrin inclusion complexes. The samples obtained in this work were subjected to DSC and XRD analyses to investigate if any crystalline drug was still present after the supercritical treatment. FTIR analyses were carried out to obtain some information about the molecular interactions between the drug and the cyclodextrin in the complexes. The drug release tests were performed to check if the formation of the drug complexes could improve the dissolution rate of the drug.

3.1. The Results of the DSC Analyses. Figure 3 reports typical DSC curves of the $\mathrm{KP}: \mathrm{Me} \beta \mathrm{CD}$ system. While the pure ketoprofen exhibits a characteristic endothermic peak at $94.5^{\circ} \mathrm{C}$, which corresponds to its melting point, the pure

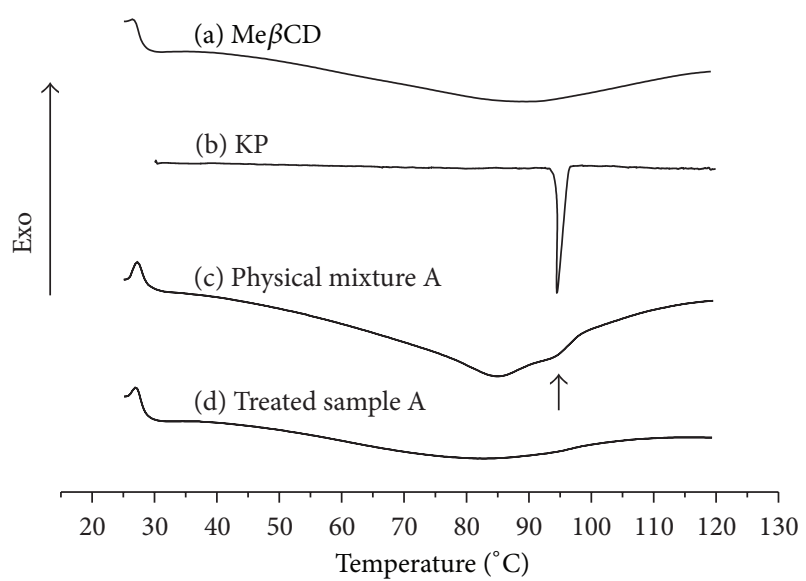

Figure 3: Typical DSC curves of the KP : Me $\beta C D$ system: (a) pure $\mathrm{Me} \beta \mathrm{CD}$, (b) pure KP, (c) physical mixture A $(16.3 \% \mathrm{KP})$, and (d) sample A $(16.3 \% \mathrm{KP})$ treated in $\mathrm{scCO}_{2}$ at $40^{\circ} \mathrm{C} 20 \mathrm{MPa} 1 \mathrm{~h}$.

Me $\beta$ CD shows a broad endothermic event between 40 and $120^{\circ} \mathrm{C}$, which corresponds to water loss.

Figure 3 also reports the DSC curves of a physical mixture and the corresponding sample after the treatment in $\mathrm{scCO}_{2}$. While the melting peak of the drug was completely absent in the treated samples, it was visible in all the physical mixtures, even though it was not very sharp and resolved. This probably occurred because the endothermic peak corresponding to the water loss of the cyclodextrin was too wide and the instrument sensitivity was too low to allow the melting peak of the KP to be clearly detected. For these reasons, only qualitative considerations can be obtained from the DSC analyses since a quantitative calculation of the crystalline drug content either in the physical mixtures or in the treated samples was not possible. It can be concluded, then, that while the physical mixtures certainly contained some amount of crystalline KP, the treated samples probably did not contain any crystalline drug, even though the instrument sensitivity did not allow its presence to be completely excluded.

3.2. The Results of the XRD Analyses. In Figure 4, the powder XRD patterns of the physical mixtures and treated samples are compared with those of the pure components. While the XRD pattern of pure KP confirms that the drug is a crystalline material, that of pure $\mathrm{Me} \beta \mathrm{CD}$ points out the amorphous character of this compound due to the presence of the two broad peaks at $11^{\circ}$ and $18^{\circ}$. As far as the physical mixtures are concerned, the XRD patterns reported in Figure 4 are simply the superimposition of those of the single components. The sharp peaks indicate the retention of the crystalline structure of the drug in the physical mixtures. On the other hand, the XRD patterns of the treated samples mainly retain the shape of that of the pure Me $\beta C D$. The disappearance of the KP diffraction patterns clearly indicates the absence of the crystalline drug in the treated samples, even though this does not allow to distinguish between drug amorphization or its inclusion into the cyclodextrin cavity. 


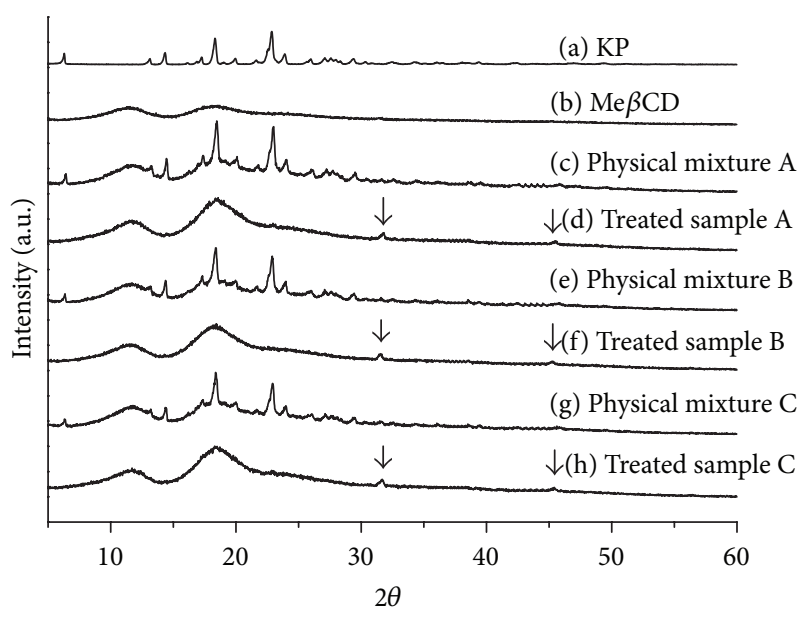

FIGURE 4: X-ray diffractograms of the pure components, their physical mixtures and the samples treated in $\mathrm{scCO}_{2}$ : (a) $\mathrm{KP}$, (b) $\mathrm{Me} \beta \mathrm{CD}$, (c) physical mixture A $(16.3 \% \mathrm{KP}),(\mathrm{d})$ treated sample A (16.3\% KP), (e) physical mixture B $(18.7 \% \mathrm{KP})$, (f) treated sample B $(18.7 \% \mathrm{KP}),(\mathrm{g})$ physical mixture C $(13.8 \% \mathrm{KP})$, and (h) treated sample C (13.8\% KP).

A closer analysis of the XRD patterns of the treated samples in Figure 4 and their comparison with that of the pure $\mathrm{Me} \beta \mathrm{CD}$ point out some changes connected to the supercritical treatment. First, the comparative intensity of the broad peaks at $11^{\circ}$ and $18^{\circ}$ was clearly modified. This phenomenon was already observable in previous works [19, $21,22]$ and was ascribed to a slight change in the structure of $\mathrm{Me} \beta \mathrm{CD}$ due to its liquefaction during the supercritical treatment [21]. Second, two peaks at $31^{\circ}$ and $45^{\circ}$ could be detected, which did not belong either to the pure KP or the pure $\mathrm{Me} \beta \mathrm{CD}$ pattern. This second phenomenon could indicate a partial recrystallization of the cyclodextrin during supercritical processing or the formation of the $\mathrm{KP} /$ cyclodextrin complex as it was, for example, suggested for the supercritical complexation of borneol and Me $\beta C D$ [21].

3.3. The Results of the FTIR Analyses. The $\mathrm{scCO}_{2}$-treated samples were analysed via FTIR spectroscopy in order to obtain further information on the physical state of the drug in the formulation and its molecular interactions with the cyclodextrin. Thus, the FTIR spectra of the physical mixtures and those of the treated samples were compared with that of the pure KP: typical spectra in the $v(\mathrm{C}=\mathrm{O})$ stretching regions are reported in Figure 5.

The pure crystalline KP exhibits two well-defined sharp peaks (Figure 5(a)): one at $1697 \mathrm{~cm}^{-1}$, representing the stretching vibration of the carbonyl group in the carboxylic acid and the other at $1655 \mathrm{~cm}^{-1}$ due to the stretching vibration of the carbonyl group in the ketonic group [30]. The former is due to the fact that, in the crystalline form, ketoprofen molecules are bound together in dimers thanks to the hydrogen bonds occurring between the carbonyl and the hydroxyl groups of the two carboxylic acids of the two molecules [35]. These two peaks can also be observed in the spectra of the

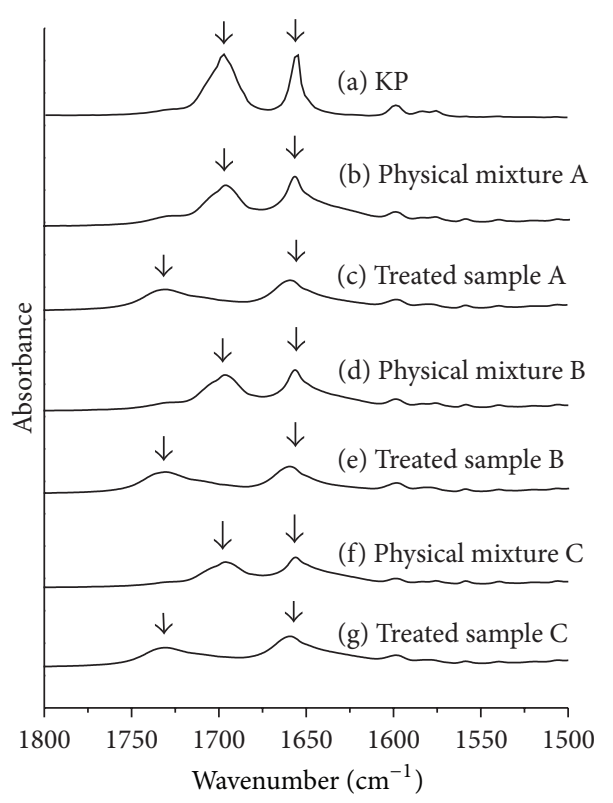

FIGURE 5: FTIR spectra curves in the $\mathrm{C}=\mathrm{O}$ stretching region of pure $\mathrm{KP}, \mathrm{KP}: \mathrm{Me} \beta \mathrm{CD}$ physical mixtures and samples treated in $\mathrm{scCO}_{2}$ at $40^{\circ} \mathrm{C}, 20 \mathrm{MPa}, 1 \mathrm{~h}$ : (a) pure $\mathrm{KP}$, (b) physical mixture A (16.3\% KP), (c) treated sample A $(16.3 \% \mathrm{KP})$, (d) physical mixture B $(18.7 \% \mathrm{KP})$, (e) treated sample B (18.7\% KP), (f) physical mixture C (13.8\% KP), and $(\mathrm{g})$ treated sample $\mathrm{C}(13.8 \% \mathrm{KP})$.

physical mixtures and, according to the literature $[35,36]$, this shows that the drug is in the crystalline form as the $\mathrm{C}=\mathrm{O}$ stretching of the dimeric ketoprofen is visible.

As far as the $\mathrm{scCO}_{2}$-treated samples is concerned, the $\mathrm{C}=\mathrm{O}$ stretching peak at $1697 \mathrm{~cm}^{-1}$ is shifted at $1730 \mathrm{~cm}^{-1}$. This shift to higher wavenumbers was already observed in a previous literature work, where $\mathrm{KP} / \mathrm{Me} \beta \mathrm{CD}$ complexation was achieved through conventional techniques [30] and was explained with the breakdown of the intermolecular hydrogen bonds between the crystalline drug molecules in the dimer and the formation of hydrogen bonds between the monomeric drug and the cyclodextrin in the complex.

3.4. The Results of the Drug Release Tests. The aim of the dissolution tests is to study the enhancement of the drug dissolution kinetics, which occurs in the treated samples. Figure 6 reports a comparison between the typical release profiles of tablets obtained from a physical mixture and the corresponding sample after the supercritical treatment. The same figure also reports the release curve for a tablet of pure KP. Both the physical mixture and the treated sample showed a significant increase in the dissolution rate with respect to the pure drug. The treated sample, then, had a faster release rate with respect to the physical mixture. While the increased dissolution rate of the physical mixtures is attributable to the in situ formation of readily soluble complexes in the dissolution medium [30], the treated samples contained "already formed" complexes, and this resulted in a further increase of the dissolution kinetics. 
TABLE 2: Indices to compare the release performance of tablets obtained from the physical mixtures and treated samples $(\mathrm{pH}=2)$.

\begin{tabular}{lccccc}
\hline & KP content $(\% \mathrm{w} / \mathrm{w})$ & $t_{50}(\mathrm{~min})$ & $t_{75}(\mathrm{~min})$ & $t_{90}(\mathrm{~min})$ & Amount of final drug release $(\%)$ \\
\hline Physical mixture A & 16.3 & 7 & 13.5 & 71 & 91 \\
Treated sample A & 16.3 & 5 & 8 & 11 & 100 \\
Physical mixture B & 18.7 & 6.5 & 11.5 & 37 & 93 \\
Treated sample B & 18.7 & 5.5 & 8.5 & 12.5 & 100 \\
Physical mixture C & 13.8 & 6 & 9.5 & 22.5 & 97 \\
Treated sample C & 13.8 & 5.5 & 8.5 & 12 & 100 \\
\hline
\end{tabular}

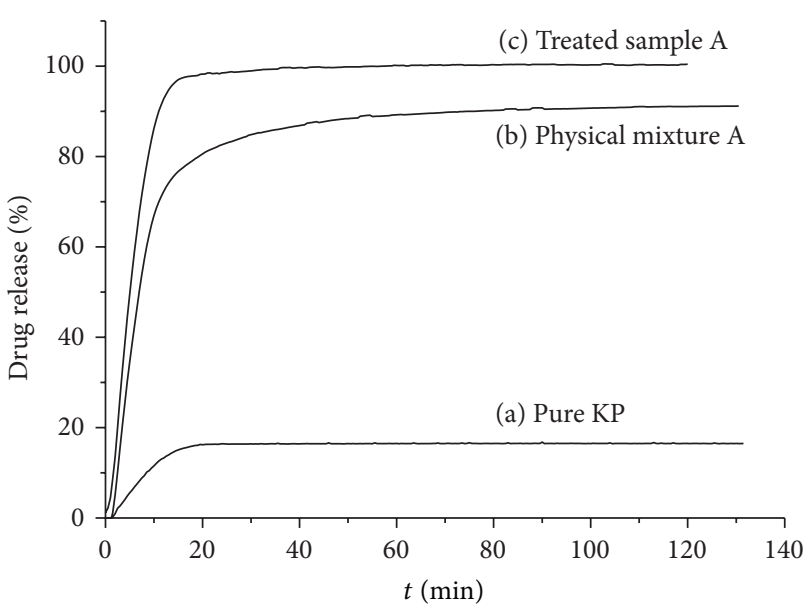

FIGURE 6: Typical percent drug release profiles of tablets in an aqueous solution at $\mathrm{pH}=2$ : (a) tablet of pure $\mathrm{KP}$, (b) $\mathrm{KP}: \mathrm{Me} \beta \mathrm{CD}$ physical mixture A (16.3\% KP), and (c) $\mathrm{KP}: \mathrm{Me} \beta \mathrm{CD}$ sample A $(16.3 \% \mathrm{KP})$ treated at $40^{\circ} \mathrm{C}, 20 \mathrm{MPa}, 1 \mathrm{~h}$.

The results of the release tests of tablets with different drug percentages are summarized in Table 2 that contains the indices $t_{50}, t_{75}$, and $t_{90}$, which are the times corresponding to $50 \%, 75 \%$, and $90 \%$ of the release, as well as the final percentage of the drug release with respect to the initial amount in the tablet, which is achieved when the steadystate conditions of the release profiles are attained. The table points out that the release rate of the treated samples was always faster than that of the physical mixtures, but this difference was significantly enhanced after the $75 \%$ of the release. Furthermore, the drug release was always complete for the treated samples while as far as the untreated samples is concerned, the same nearly occurred only for the physical mixture $\mathrm{C}$, where the drug content was in defect with respect to the stoichiometric ratio. Even though the supercritical complexation is beneficial to the dissolution kinetics for all drug contents, the best improvement was found for the samples where the KP and the $\mathrm{Me} \beta C D$ were in equimolar ratio (A). The $90 \%$ of the drug release, in fact, was attained after $11 \mathrm{~min}$ by the treated samples, while 71 minutes were required by the physical mixtures. This confirms the recommendation by Mura and coworkers [30] who suggested a 1:1 stoichiometric ratio between the drug and the cyclodextrin to obtain inclusion complexes through conventional techniques.
TABLE 3: Values of the $k$ parameter obtained from the fitting of the release profiles of tablets to (1).

\begin{tabular}{lcc}
\hline & KP content $(\% \mathrm{w} / \mathrm{w})$ & $k\left(\mathrm{~min}^{-1}\right)$ \\
\hline Physical mixture A & 16.3 & 0.153 \\
Treated sample A & 16.3 & 0.227 \\
Physical mixture B & 18.7 & 0.165 \\
Treated sample B & 18.7 & 0.225 \\
Physical mixture C & 13.8 & 0.187 \\
Treated sample C & 13.8 & 0.224 \\
\hline
\end{tabular}

The release profiles of the tablets were also normalized with respect to the steady-state drug concentration in the aqueous solution and fitted to the following simple model [37]:

$$
\frac{C}{C_{\infty}}=1-e^{-k t}
$$

The values of the release coefficient $k$ obtained for the treated samples are compared with those of the corresponding physical mixtures in Table 3 . The data confirm that the highest gain in the release rate was obtained for the treated sample A.

Since the "dispersed amount method" is generally preferred to the "tablet method" in many previous literature works $[12,15,16,19,24,30]$, other release tests were carried out directly using the powders of the physical mixtures or the treated samples without producing any tablets. Table 4 reports the release indices obtained for the equimolar samples (A), but similar results were also obtained for the other drug contents. These tests showed that the release profiles of the treated samples were almost instantaneous due to the huge increase in the mass transfer area of the powders with respect to the tablets. On the other hand, the dissolution kinetics was too fast to allow any difference in the release rate of the samples with different drug contents to be clearly distinguished.

Eventually, some release tests were performed to investigate if the drug release was affected by the value of the $\mathrm{pH}$ as it occurred, for example, in the ibuprofen/ $\beta$-cyclodextrin system [24]. The results showed that even though the release rates of the physical mixtures were moderately affected by the $\mathrm{pH}$ value, those of the treated samples were completely unaffected by this parameter (Figure 7). 
TABLE 4: Indices to compare the release performance of powders of the physical mixtures and treated samples $(\mathrm{pH}=2)$.

\begin{tabular}{lccccc}
\hline & KP content $(\% \mathrm{w} / \mathrm{w})$ & $t_{50}(\mathrm{~min})$ & $t_{75}(\mathrm{~min})$ & $t_{90}(\mathrm{~min})$ & Amount of final drug release $(\%)$ \\
\hline Physical mixture A & 16.3 & 1 & 12 & 27 & 99 \\
Treated sample A & 16.3 & $<1$ & $<1$ & 1 & 100 \\
\hline
\end{tabular}

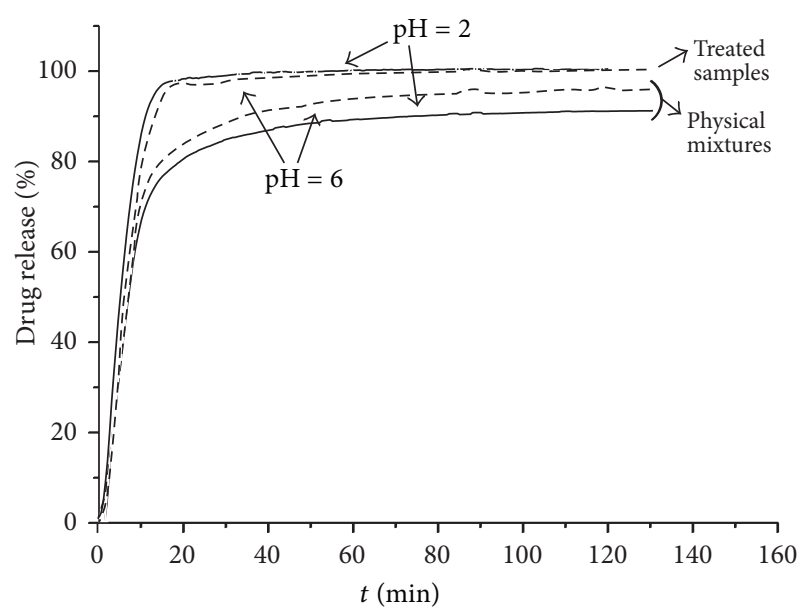

Figure 7: Comparison between the percent drug release profiles of tablets in an aqueous solution at different $\mathrm{pH}$ values (solid line: $\mathrm{pH}=2$; dashed line: $\mathrm{pH}=6)$. The tablets were obtained from physical mixture A $(16.3 \% \mathrm{KP})$ and sample A $(16.3 \% \mathrm{KP}$, treated at $\left.40^{\circ} \mathrm{C}, 20 \mathrm{MPa}, 1 \mathrm{~h}\right)$.

\section{Conclusion}

This work has shown that $\mathrm{scCO}_{2}$ can be an efficient method to obtain inclusion complexes of $\mathrm{KP}$ with $\mathrm{Me} \beta \mathrm{CD}$. The supercritical process was investigated with this drug/cyclodextrin system for the first time. The choice of $\mathrm{Me} \beta \mathrm{CD}$ as the host cyclodextrin molecule is particularly attractive since this compound is melted during the supercritical process, and this favours the complexation step without any addition of water or auxiliary agents.

Samples at different KP contents were prepared and the DSC, XRD, and FTIR characterization allowed exclude the presence of crystalline drug and hypothesise the formation of the complexes. The drug release tests showed that the dissolution rate of the supercritical complexes was improved both with respect to the pure drug and the physical mixtures.

The powder or tablet formulation of the drug complexes during the in vitro release tests influenced the role of the drug amount in the samples. While the dispersed release of the powders was almost instantaneous regardless of the drug content, the highest improvement in the dissolution kinetics was found for a sample that contained equimolar amounts of both the drug and the cyclodextrin in the tablet. On the other hand, a $\mathrm{pH}$ variation of the dissolution medium from 2 to 6 did not show any effect on the release rate of the supercritical complexes that are, then, expected to offer the same bioavailability of the drug all along the gastrointestinal tract.

\section{Conflict of Interests}

The authors declare no conflict of interests.

\section{References}

[1] P. Girotra, S. K. Singh, and K. Nagpal, "Supercritical fluid technology: a promising approach in pharmaceutical research," Pharmaceutical Development and Technology, vol. 18, no. 1, pp. 22-38, 2013.

[2] I. Pasquali and R. Bettini, "Are pharmaceutics really going supercritical?" International Journal of Pharmaceutics, vol. 364, no. 2, pp. 176-187, 2008.

[3] B. S. Sekhon, "Supercritical fluid technology: an overview of pharmaceutical applications," International Journal of PharmTech Research, vol. 2, no. 1, pp. 810-826, 2010.

[4] T. Loftsson and M. E. Brewster, "Pharmaceutical applications of cyclodextrins. 1. Drug solubilization and stabilization," Journal of Pharmaceutical Sciences, vol. 85, no. 10, pp. 1017-1025, 1996.

[5] T. Loftsson, "Pharmaceutical applications of $\beta$-cyclodextrin," Pharmaceutical Technology, vol. 23, no. 12, pp. 40-50, 1999.

[6] T. Sultana, J. M. Jung, S. S. Hong et al., "Characteristic profiles of the inclusion complex of omeprazole/peracylated- $\beta$ cyclodextrin formed in supercritical carbon dioxide," Journal of Inclusion Phenomena and Macrocyclic Chemistry, vol. 72, no. 1-2, pp. 207-207, 2012.

[7] R. L. Carrier, L. A. Miller, and I. Ahmed, "The utility of cyclodextrins for enhancing oral bioavailability," Journal of Controlled Release, vol. 123, no. 2, pp. 78-99, 2007.

[8] M. E. Brewster and T. Loftsson, "Cyclodextrins as pharmaceutical solubilizers," Advanced Drug Delivery Reviews, vol. 59, no. 7, pp. 645-666, 2007.

[9] S. Y. Lee, I. I. Jung, J. K. Kim, G. B. Lim, and J. H. Ryu, "Preparation of itraconazole/HP- $\beta$-CD inclusion complexes using supercritical aerosol solvent extraction system and their dissolution characteristics," Journal of Supercritical Fluids, vol. 44, no. 3, pp. 400-408, 2008.

[10] T. van Hees, G. Piel, B. Evrard, X. Otte, L. Thunus, and L. Delattre, "Application of supercritical carbon dioxide for the preparation of a piroxicam- $\beta$-cyclodextrin inclusion compound," Pharmaceutical Research, vol. 16, no. 12, pp. 1864-1870, 1999.

[11] T. Van Hees, V. Barillaro, G. Piel et al., "Application of supercritical carbon dioxide for the preparation of drug-cyclodextrin inclusion compounds," Journal of Inclusion Phenomena, vol. 44, no. 1-4, pp. 271-274, 2002.

[12] M. Moneghini, I. Kikic, B. Perissutti, E. Franceschinis, and A. Cortesi, "Characterisation of nimesulide-betacyclodextrins systems prepared by supercritical fluid impregnation," European Journal of Pharmaceutics and Biopharmaceutics, vol. 58, no. 3, pp. 637-644, 2004.

[13] A. H. Al-Marzouqi, I. Shehatta, B. Jobe, and A. Dowaidar, "Phase solubility and inclusion complex of itraconazole with 
$\beta$-cyclodextrin using supercritical carbon dioxide," Journal of Pharmaceutical Sciences, vol. 95, no. 2, pp. 292-304, 2006.

[14] A. Bounaceur, E. Rodier, and J. Fages, "Maturation of a ketoprofen/ $\beta$-cyclodextrin mixture with supercritical carbon dioxide," Journal of Supercritical Fluids, vol. 41, no. 3, pp. 429-439, 2007.

[15] A. Al-Marzouqi, B. Jobe, G. Corti, M. Cirri, and P. Mura, "Physicochemical characterization of drug-cyclodextrin complexes prepared by supercritical carbon dioxide and by conventional techniques," Journal of Inclusion Phenomena and Macrocyclic Chemistry, vol. 57, no. 1-4, pp. 223-231, 2007.

[16] M. Sauceau, E. Rodier, and J. Fages, "Preparation of inclusion complex of piroxicam with cyclodextrin by using supercritical carbon dioxide," Journal of Supercritical Fluids, vol. 47, no. 2, pp. 326-332, 2008.

[17] A. H. Al-Marzouqi, A. Solieman, I. Shehadi, and A. Adem, "Influence of the preparation method on the physicochemical properties of econazole- $\beta$-cyclodextrin complexes," Journal of Inclusion Phenomena and Macrocyclic Chemistry, vol. 60, no. 12, pp. 85-93, 2008.

[18] A. H. Al-Marzouqi, H. M. Elwy, I. Shehadi, and A. Adem, "Physicochemical properties of antifungal drug-cyclodextrin complexes prepared by supercritical carbon dioxide and by conventional techniques," Journal of Pharmaceutical and Biomedical Analysis, vol. 49, no. 2, pp. 227-233, 2009.

[19] M. Charoenchaitrakool, F. Dehghani, and N. R. Foster, "Utilization of supercritical carbon dioxide for complex formation of ibuprofen and methyl- $\beta$-cyclodextrin," International Journal of Pharmaceutics, vol. 239, no. 1-2, pp. 103-112, 2002.

[20] N. Bandi, W. Wei, C. B. Roberts, L. P. Kotra, and U. B. Kompella, "Preparation of budesonide- and indomethacinhydroxypropyl-beta-cyclodextrin (HPBCD) complexes using a single-step, organic-solvent-free supercritical fluid process," European Journal of Pharmaceutical Sciences, vol. 23, no. 2, pp. 159-168, 2004.

[21] J. He and W. Li, "Preparation of borneol-methyl- $\beta$-cyclodextrin inclusion complex by supercritical carbon dioxide processing," Journal of Inclusion Phenomena and Macrocyclic Chemistry, vol. 65, no. 3, pp. 249-256, 2009.

[22] J. He, "Complex between modified $\beta$-cyclodextrins and three components of traditional Chinese medicine in supercritical carbon dioxide medium," Journal of Inclusion Phenomena and Macrocyclic Chemistry, vol. 68, no. 3-4, pp. 399-410, 2010.

[23] M. Banchero and L. Manna, "Investigation of the piroxicam/ hydroxypropyl- $\beta$-cyclodextrin inclusion complexation by means of a supercritical solvent in the presence of auxiliary agents," Journal of Supercritical Fluids, vol. 57, no. 3, pp. 259-266, 2011.

[24] M. Türk, G. Upper, M. Steurenthaler, K. Hussein, and M. A. Wahl, "Complex formation of Ibuprofen and $\beta$-Cyclodextrin by controlled particle deposition (CPD) using $\mathrm{SC}-\mathrm{CO}_{2}$," Journal of Supercritical Fluids, vol. 39, no. 3, pp. 435-443, 2007.

[25] S. W. Jun, M. S. Kim, J. S. Kim et al., "Preparation and characterization of simvastatin/hydroxypropyl- $\beta$-cyclodextrin inclusion complex using supercritical antisolvent (SAS) process," European Journal of Pharmaceutics and Biopharmaceutics, vol. 66, no. 3, pp. 413-421, 2007.

[26] V. Barillaro, P. Bertholet, S. H. de Hassonville et al., "Effect of acidic ternary compounds on the formation of miconazole/ cyclodextrin inclusion complexes by means of supercritical carbon dioxide," Journal of Pharmacy and Pharmaceutical Sciences, vol. 7, no. 3, pp. 378-388, 2004.
[27] M. Banchero and L. Manna, "The use of lysine to enhance the supercritical complexation of ketoprofen and cyclodextrins," Journal of Supercritical Fluids, vol. 67, pp. 76-83, 2012.

[28] H. E. Grandelli, J. C. Hassler, A. Whittington, and E. Kiran, "Melting point depression of Piroxicam in carbon dioxide + co-solvent mixtures and inclusion complex formation with $\beta$ cyclodextrin," Journal of Supercritical Fluids, vol. 71, pp. 19-25, 2012.

[29] P. Mura, G. P. Bettinetti, A. Manderioli, M. T. Faucci, G. Bramanti, and M. Sorrenti, "Interactions of ketoprofen and ibuprofen with $\beta$-cyclodextrins in solution and in the solid state," International Journal of Pharmaceutics, vol. 166, no. 2, pp. 189-203, 1998.

[30] P. Mura, M. T. Faucci, P. L. Parrini, S. Furlanetto, and S. Pinzauti, "Influence of the preparation method on the physicochemical properties of ketoprofen-cyclodextrin binary systems," International Journal of Pharmaceutics, vol. 179, no. 1, pp. 117-128, 1999.

[31] M. Cirri, F. Maestrelli, N. Mennini, and P. Mura, "Physicalchemical characterization of binary and ternary systems of ketoprofen with cyclodextrins and phospholipids," Journal of Pharmaceutical and Biomedical Analysis, vol. 50, no. 5, pp. 683689, 2009.

[32] M. Banchero, L. Manna, S. Ronchetti, P. Campanelli, and A. Ferri, "Supercritical solvent impregnation of piroxicam on PVP at various polymer molecular weights," Journal of Supercritical Fluids, vol. 49, no. 2, pp. 271-278, 2009.

[33] M. Banchero, A. Ferri, and L. Manna, "The phase partition of disperse dyes in the dyeing of polyethylene terephthalate with a supercritical $\mathrm{CO}_{2}$ /methanol mixture," Journal of Supercritical Fluids, vol. 48, no. 1, pp. 72-78, 2009.

[34] M. Banchero, A. Ferri, L. Manna, and S. Sicardi, "Solubility of disperse dyes in supercritical carbon dioxide and ethanol," Fluid Phase Equilibria, vol. 243, no. 1-2, pp. 107-114, 2006.

[35] L. Manna, M. Banchero, D. Sola, A. Ferri, S. Ronchetti, and S. Sicardi, "Impregnation of PVP microparticles with ketoprofen in the presence of supercritical $\mathrm{CO}_{2}$," Journal of Supercritical Fluids, vol. 42, no. 3, pp. 378-384, 2007.

[36] S. G. Kazarian and G. G. Martirosyan, "Spectroscopy of polymer/drug formulations processed with supercritical fluids: in situ ATR-IR and Raman study of impregnation of ibuprofen into PVP," International Journal of Pharmaceutics, vol. 232, no. 1-2, pp. 81-90, 2002.

[37] P. Costa and J. M. Sousa Lobo, "Modeling and comparison of dissolution profiles," European Journal of Pharmaceutical Sciences, vol. 13, no. 2, pp. 123-133, 2001. 

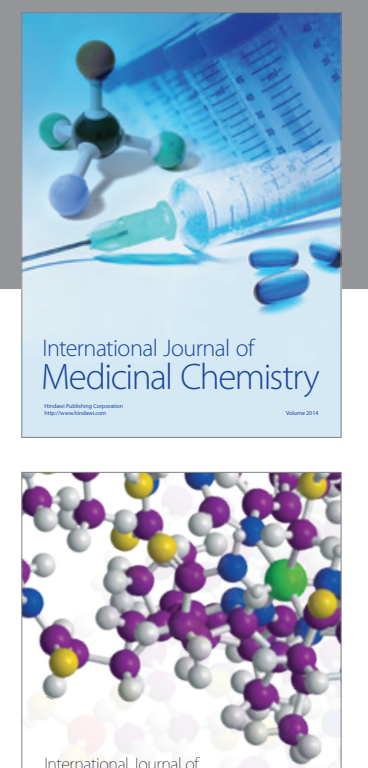

\section{Carbohydrate} Chemistry

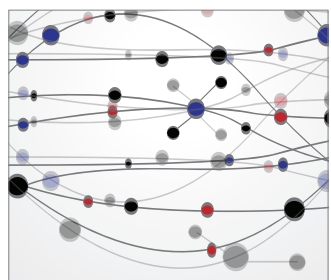

The Scientific World Journal
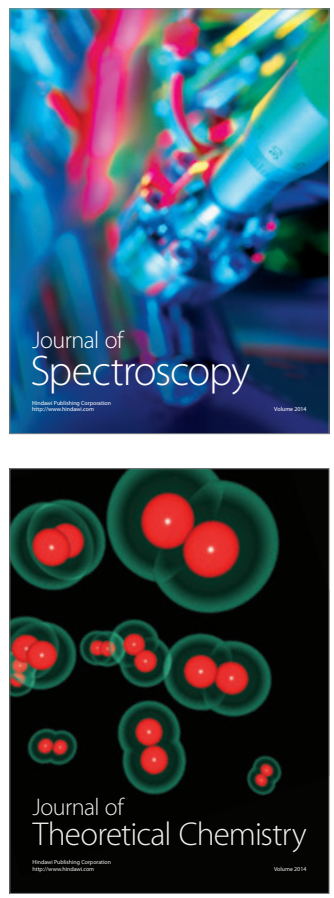
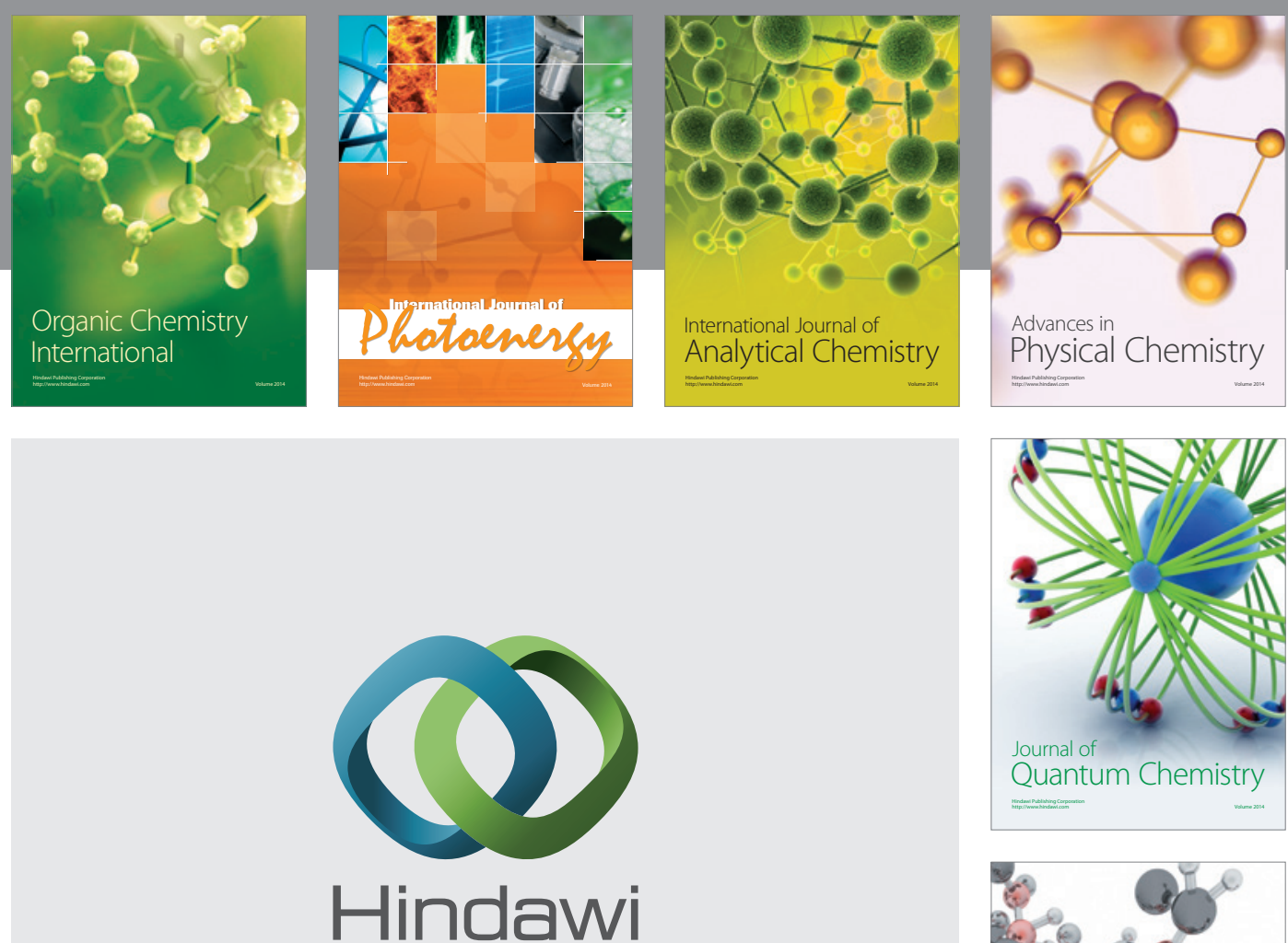

Submit your manuscripts at

http://www.hindawi.com

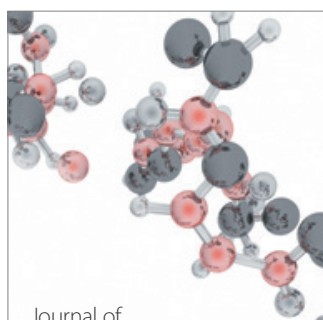

Analytical Methods

in Chemistry

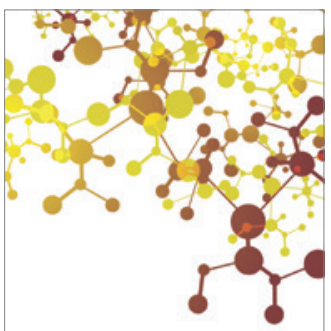

Journal of

Applied Chemistry

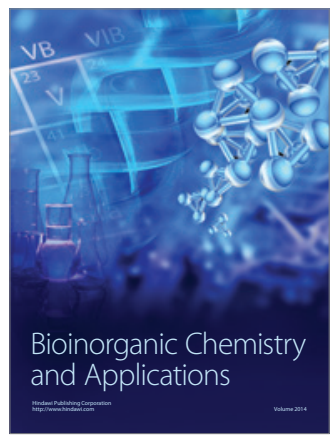

Inorganic Chemistry
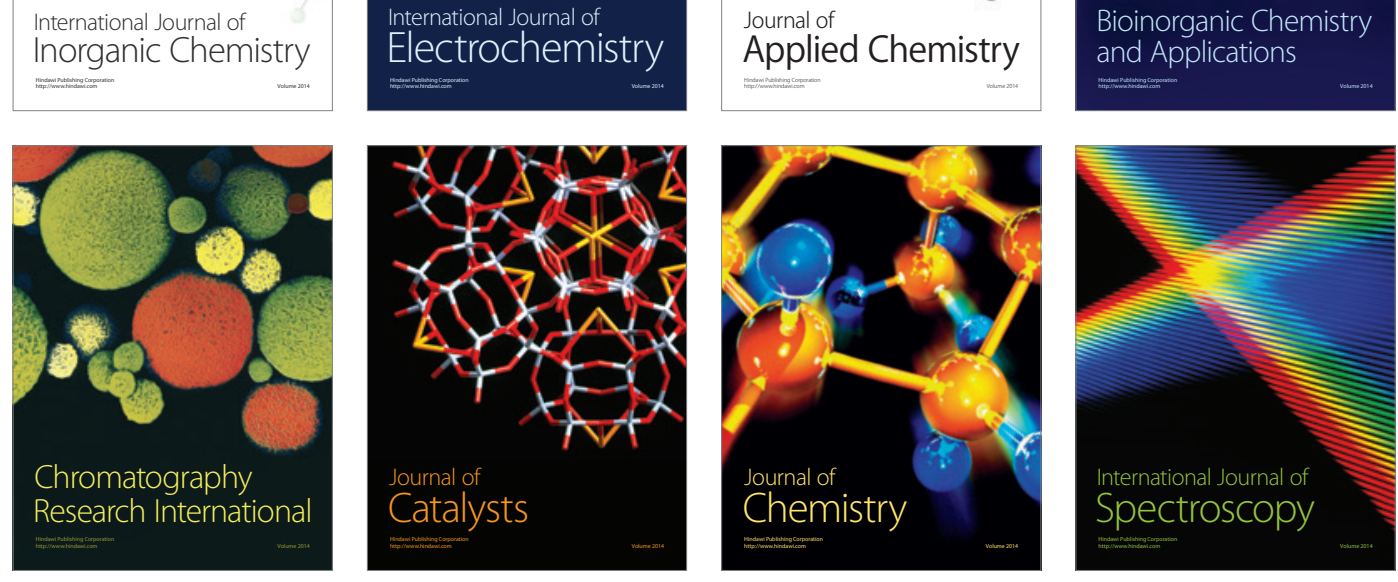